\title{
Utilizing Drones to Restore and Maintain Radio Communication During Search and Rescue Operations
}

\author{
Jake N. McRae, BSN; Brandon M. Nielsen, BS; Christopher J. Gay, MPH; Andrew P. Hunt, MBS; \\ Andrew D. Nigh, MD \\ Rocky Vista University College of Osteopathic Medicine, Ivins, UT
}

\begin{abstract}
Introduction-The ability of rescuers to maintain contact with incident command (IC) and each other is a critical component of search and rescue (SAR) operations. When rescuers lose radio communication with operation leaders, the effectiveness of operations may be substantially affected. This often occurs owing to the limitations of standard communications equipment in difficult terrain or when victims are beyond line-of-sight. This study investigates the viability of using an aerial drone-repeater system configuration to restore and maintain radio communications between IC and deployed rescuers.

Methods-SAR operators in Southern Utah identified 10 areas where radio communication is compromised during live rescue operations. Trained SAR personnel were deployed to these areas in a mock exercise. After confirmed loss of communication, a repeater-equipped aerial drone was piloted $122 \mathrm{~m}$ above IC to restore communication. Once restored, communication was assessed at regular intervals for the duration of the mock deployment.

Results-In all 10 areas tested, communication was successfully restored. In all cases, once communication was restored, no additional loss of radio contact occurred. The time between communication loss and restoration across the 10 scenarios was $6.5 \pm 1.1$ (4.4-9.3) min (mean $\pm \mathrm{SD}$ with range).

Conclusions - This method of restoring radio communication among SAR personnel could drastically improve the ability to assist victims and help mitigate the risks faced by rescuers. SAR leaders should be made aware of the useful applications of drones during SAR operations, especially in instances where communication is compromised.
\end{abstract}

Keywords: mountain rescue, unmanned aerial vehicle, wilderness medicine

\section{Introduction}

Backcountry activities have become more popular in the most recent decade, potentially leading to an increase in search and rescue (SAR) cases and deployments. ${ }^{1,2}$ Although the advancement of technology has made the outdoors safer to navigate, the ability of rescuers in the field to reliably communicate with incident command (IC) during live SAR operations has been slower to develop. This is problematic when victims are lost or stranded in remote locations and difficult terrain.

The ability of rescuers to maintain contact with IC is one of the most critical components of any SAR

Corresponding author: Jake N. McRae, BSN, Rocky Vista University, College of Osteopathic Medicine, 332 Ridge Road, Orem, UT 84057; e-mail: jakeneilmcrae@gmail.com.

Submitted for publication April 2020.

Accepted for publication November 2020. operation. ${ }^{3}$ The typical communication equipment library of regional SAR teams consists of 5-8 W dual-band 2way radios. ${ }^{4}$ Given the available equipment, it is possible for deployed SAR personnel to lose radio communication with operation leaders. ${ }^{4}$ When communication loss occurs during a SAR event, it has a significant impact on the ability of rescue personnel to request additional resources, deliver medical aid, relay details relative to victim status, or call for an emergency evacuation. ${ }^{4}$ This issue is often a substantial concern owing to the nature of the terrain where operations frequently take place, such as in canyons or mountains where victims are likely located beyond line-of-sight. ${ }^{4}$

To maintain the safety of mobilized SAR personnel and victims, reliable communication during live SAR operations is critical in cases involving medical or traumatic circumstances and in the implementation of largescale search operations. According to several SAR 
epidemiologic studies conducted in the United States, Canada, and New Zealand covering thousands of SAR incidents between 1970 and 2017, the percentage of SAR rescues that involve medical and trauma victims ranges from 31 to $89 \%$ annually. ${ }^{5-9}$ In Canadian national parks and Yosemite National Park, investigations indicated emergent helicopter evacuation and transport in 64 and $28 \%$ of cases, respectively. ${ }^{6,10}$ This highlights the need for rescuers and SAR operators to maintain a reliable channel of communication because communication loss is likely to lead to delays in the delivery of medical aid to victims, decrease the chances of successful search or nonfatal rescue, ${ }^{4}$ and increase the risk placed upon rescuers. The consequences of communication issues can be made worse in more urgent circumstances where large-scale search operations require constant communication and coordination between multiple search teams.

Recently, there has been an increased application of unmanned aerial vehicles, or drones, for SAR purposes. ${ }^{11-13}$ For example, aerial drones have been successfully used to locate $^{14}$ and deliver aid to victims $^{11}$ in specific situations where rescuers were delayed or there was great risk to their safety. The objective of this study was to investigate the viability of using an aerial drone equipped with a lightweight portable simplex repeater system to restore and maintain radio communications between IC and SAR personnel in terrain where radio communication loss is known to occur.

\section{Methods}

Relevant ethical safeguards were appropriately met in relation to subject protection, and the study was approved by the Rocky Vista University institutional review board. Southern Utah SAR operators were consulted to identify areas in the region where radio communications are commonly compromised during live SAR events. For a location to be included in the investigation, several criteria had to be met: The site must have been the location of a live rescue event in the last $3 \mathrm{y}$, radio communication between rescuers and IC must have been lost or compromised during the operation without reestablishment of communication at the location or in the immediate surrounding area of the victim, attempts were made to reestablish communication but failed, the cause of the communication loss must have been likely due to terrain characteristics, and global positioning system coordinates of IC and victim were recorded. Ten sites across southern Utah were identified and confirmed to have terrain characteristics that likely contributed to communication loss. The terrain present at the identified locations included slot canyons, vertical cliffs, steep mountains, and wide deserts (Figure 1).

The primary objective of the experiment was to determine whether communication could be restored at each of the 10 sites using a drone equipped with a lightweight simplex repeater system. In a simulation, a team of 2 formally trained SAR personnel was deployed to each of the 10 identified locations. SAR team member 1, a certified Part 107 drone pilot referred to as "IC," was stationed at the coordinates corresponding to IC during the live event and was equipped with a BaoFeng BFF8HP $5 \mathrm{~W}$ 2-way radio consistent with the equipment regularly employed by regional $\mathrm{SAR},{ }^{4}$ as well as the experimental configuration of a Mavic 2 aerial drone with a tether-mounted Surecom SR-112 controller-repeater system (Figure 2). SAR team member 2, referred to as "rescuer," traveled on foot, equipped with a BaoFeng BFF8HP 5 W 2-way radio comparable to those widely used in SAR operations, ${ }^{4}$ to the coordinates where the victim was encountered.

The radio frequencies used during the investigation are frequencies reserved for use by SAR personnel and emergency responders in the state of Utah. Relevant permissions were obtained from the Washington County sheriff's office for their use in the study.

A complete loss of communication was characterized as total silence during attempted radio transmission. Before radio silence and subsequent drone-repeater system deployment, communication between IC and rescuer was clear: The rescuer could easily understand and be understood by IC, and vice versa. Once loss of communication between team members was confirmed, often after rescuer entry into a canyon or movement beyond a ridge or hill, IC deployed the drone-repeater system configuration. The drone was piloted $122 \mathrm{~m}$ directly, or nearly directly, above IC in the attempt to restore communication. Once communication was restored, it was assessed every $2 \mathrm{~min}$ for the duration of the

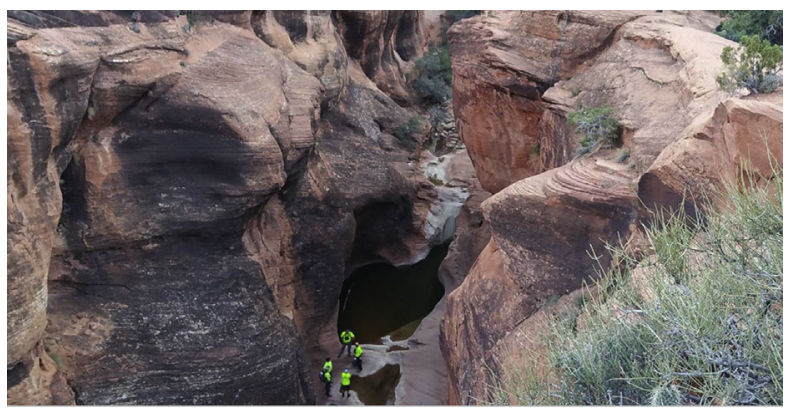

Figure 1. Example of terrain where communication loss occurred. Photo courtesy of Washington County Search and Rescue Team. 


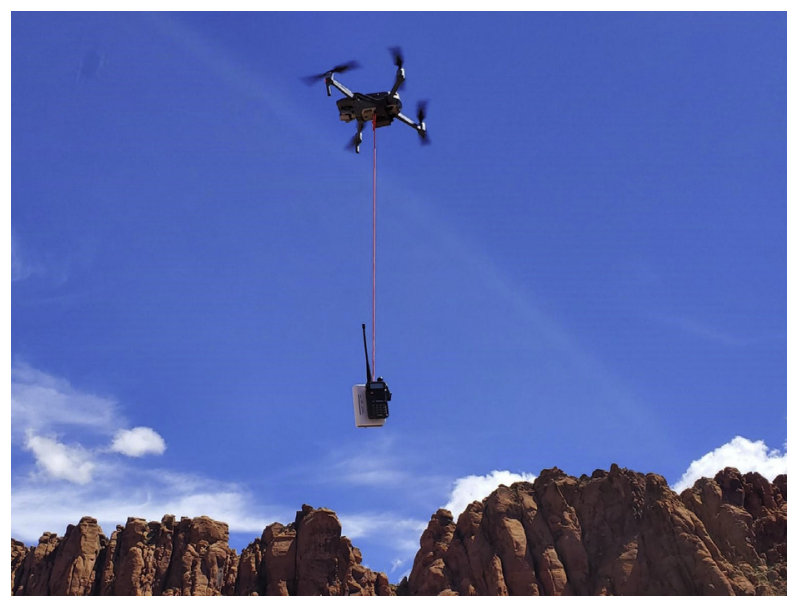

Figure 2. Surecom SR-112 controller-repeater equipped to an aerial drone (Mavic 2 Pro). Photo courtesy of Washington County Search and Rescue Team.

personnel deployment from the point of loss of communication to the location of the victim and back to IC. A single drone battery charge enables $18 \mathrm{~min}$ of continuous drone use. To allow for the continual service of the drone, a battery bank consisting of 3 standard Mavic 2 drone batteries and a mobile charging station were used throughout the experiment. Global positioning system coordinates and communication status were recorded.

An additional objective of the experiment was to assess the amount of time elapsed between communication loss and restoration to provide an approximation of the efficiency of the drone-repeater system configuration in communication reestablishment. Times are presented as mean $\pm \mathrm{SD}$ with range.

\section{Results}

Clear radio communication was lost and subsequently restored in all 10 of the tested locations (Table 1). In 9 out of 10 locations, communication was lost completely. In 1 location, consisting of mostly rolling sand dune type terrain, communication was reduced to a faint uniform static lacking any decipherable form of radio transmission. After the deployment of the drone-repeater system configuration, radio communication was restored to baseline in all 10 locations and allowed for the simulated IC and search team to exchange dialogue with minimal, if any, static or interruption. Across all 10 scenarios, the time elapsed from communication loss to restoration was 6.5 $\pm 1.1(4.4-9.9) \mathrm{min}$ (Table 1). In every case, after reestablishment of communication, IC and the rescuer maintained contact throughout the remainder of the simulated operation.
In 4 of the 10 locations, the drone-repeater system was unable to restore radio communication after being flown directly above IC. In these cases, the drone was piloted horizontally in the direction of the rescuer, and clear communication was subsequently achieved. At no point during the experiment did the drone need to fly out of line-of-sight from the drone pilot before restoring communication between IC and rescuer. The maximum horizontal distance necessary for the drone to travel to reestablish communication was $115 \mathrm{~m}$ (Table 1).

In most cases, communication between the SAR team members was lost at the same time regardless of which member initiated the transmission. Occasionally, IC could hear a transmission from the rescuer, but could not be heard in a return transmission for a brief period before complete communication loss was noted.

\section{Discussion}

The locations examined in this study had a diverse terrain characteristics, including mountains, sand dunes, and slot canyons. Because most land-based SAR operations take place in wilderness areas consisting of mountainous environments, this method of reestablishing communication may be applied broadly to wilderness SAR teams. SAR leaders should be made aware that drones equipped with repeater systems can be a cost-effective way to recover radio communication that is lost owing to difficult terrain topography. Giving SAR teams the capability to maintain communications via use of drones could potentially result in more efficient execution of operations and promote better outcomes for victims requiring urgent aid and/or transport.

Our investigation demonstrates that drones with attached repeater systems can be a simple, effective, and relatively low-cost solution to incidents of radio communication inconsistency or loss among SAR personnel. Radios are the primary mode of communication used in SAR operations. When radios fail to allow adequate communication, cell phones are often used as a replacement. However, if cell phone reception is not available, as is the case in most wilderness settings, the options for restoring communication between IC and SAR teams become limited to devices that communicate via satellite or radio repeaters. The Mavic 2 drone and Surecom SR-112 controller-repeater system configuration used in this study retail for around \$1350 and \$55 USD, respectively. ${ }^{4,15}$ This equipment should be adequate for most SAR teams to achieve the same performance demonstrated in our investigation. However, SAR teams can expect cost to rise should they invest in additional accessories, higher-performance drones, more powerful 
Table 1. Data collected from mock search and rescue scenarios

\begin{tabular}{llllll}
\hline Location & $\begin{array}{l}\text { Distance between } \\
\text { IC and victim } \\
(\mathrm{km})\end{array}$ & $\begin{array}{l}\text { Communication } \\
\text { restored and } \\
\text { maintained? }\end{array}$ & $\begin{array}{l}\text { Radio status at } \\
\text { communication } \\
\text { loss }\end{array}$ & $\begin{array}{l}\text { Horizontal distance } \\
\text { flown to restore } \\
\text { communication }(m)\end{array}$ & $\begin{array}{l}\text { Time elapsed between } \\
\text { communication loss } \\
\text { and restoration }(\text { min:s) }\end{array}$ \\
\hline 1 & 1.29 & Yes & Silent & 0 & $5: 14$ \\
2 & 4.02 & Yes & Silent & 87 & $8: 37$ \\
3 & 5.63 & Yes & Static & 63 & $7: 43$ \\
4 & 0.87 & Yes & Silent & 0 & $4: 27$ \\
5 & 1.03 & Yes & Silent & 0 & $5: 42$ \\
6 & 5.95 & Yes & Silent & 68 & $8: 43$ \\
7 & 2.74 & Yes & Silent & 0 & $5: 22$ \\
8 & 3.70 & Yes & Silent & 0 & $4: 56$ \\
9 & 8.85 & Yes & Silent & 115 & $9: 18$ \\
10 & 3.38 & Yes & Silent & 0 & $5: 11$ \\
\hline
\end{tabular}

repeater systems, and longer-lasting batteries. Satellite phones and similar devices may be considered, but they come with unique limitations and costs and commonly require service subscriptions.

The results of our study indicate that this method of reestablishing communication among SAR personnel has the potential to improve wilderness medical outcomes and prevent fatalities. A reliable line of communication allows SAR medical teams to request vital supplies, additional personnel, and helicopter evacuation. As highlighted previously, a significant proportion of SAR cases require the use of medical treatment, especially in cases of trauma. With the complexity of coordinating medical care and victim transport in wilderness environments, consistent and clear communication is paramount.

Although the recorded time intervals between communication loss and restoration showed that in all cases communication was restored in less than $10 \mathrm{~min}$, the SAR team prioritized confirmed reestablishment of communication over how quickly communication was restored. Thus, it is reasonable to assert that this could be done more efficiently. We hypothesize that the use of drones to restore radio communication will decrease the average time it takes for victims to receive vital medical care and transport, which may result in decreased morbidity and mortality. However, additional data and investigation of the live application of drones in this context will be needed to support this.

For SAR teams considering the tested configuration as a potential solution, some important aspects should be considered. First, SAR teams should research local and regional drone regulations, as specific laws may limit where or how a drone may be used for this application. Second, a drone battery bank should be strongly considered. If SAR teams invest in multiple drone batteries and a mobile charging station, they will have the ability to quickly replace an exhausted battery with a freshly charged battery and return the drone to service. For the drone used in this study, 3 batteries are the minimum number required for continual drone use, and each battery provided roughly $18 \mathrm{~min}$ of flight time.

\section{LIMITATIONS}

The effectiveness of the drone-repeater system configuration to restore communication in every location that was tested should reassure SAR teams that there is a feasible solution for scenarios in which radio communication is lost due to rugged terrain; however, users should be aware of specific limitations.

In our study, the longest distance between IC and the deployed SAR team was $8.9 \mathrm{~km}$, and communication was restored with a single SAR team in each case (Table 1). In instances where SAR operations cover large distances and multiple teams, it is possible that additional or more powerful equipment would be needed to restore communication. More strategic placement of the drone and pilot might also be considered (ie, between IC and the victim). Teams that want the option to have their pilot travel on foot to a more favorable location should consider drones that are lightweight and mobile, such as the one used in this study.

It should also be mentioned that the study worked within the limitations of line-of-sight laws, which require drone pilots to keep the drone in their line-of-sight. ${ }^{16}$ If a waiver is obtained, or if the pilot is operating in an area where these laws do not apply, it is possible that communication could be restored in more complicated or restrictive terrain. ${ }^{16}$ Drone operators and SAR teams should assess the limitations of drone laws before they implement drones equipped with repeaters. We recommend that SAR leaders become familiar with local laws regarding the use of repeaters and the frequencies 
available for their use, as there may be legal authorization considerations in placing a mobile repeater on public health frequencies. ${ }^{17}$ We suspect that SAR teams could use the radio frequencies that they already use, but it is important to confirm this before committing resources to this potential solution.

A simplex repeater uses a single radio frequency to transmit and receive communication, requiring the transmitting user to complete an entire communication before it is relayed to the receiving user. ${ }^{18} \mathrm{~A}$ traditional duplex repeater transmits and receives on different frequencies, allowing simultaneous transmission and receiving. ${ }^{18}$ The simplex repeater was chosen for this study owing to its simplicity in equipment setup and use, although it has its disadvantages. Owing to nonsimultaneous transmission and receiving, it requires a delay after transmission for the repeat. Increased orientation and training are required for use of simplex repeaters, in which the SAR personnel participating in the investigation were proficient, to mitigate the delay and repeat in transmission.

Although the communications equipment used in this study was standard for SAR operators in southern Utah, we recognize that different equipment may be used by other SAR teams in other regions. This may have affected the timing and location of communication loss and restoration when compared to equipment used elsewhere. However, we would not anticipate a significant variation in the performance of the equipment used in this study and standard equipment used by other SAR teams around the world.

In addition to following drone regulations, SAR teams should make substantial efforts to comply with the Federal Communications Commission (FCC) radio communication and frequency use regulations and restrictions; there are specific authorizations and equipment performance specifications to operate on emergency frequencies. It is important to note that although the equipment used in the experiment is commonly employed by SAR teams, specific radio models, such as those manufactured by BaoFeng, are controversial with respect to their legal approval by the FCC for use on public health frequencies. Equipment specifications are important to keep in mind because they include details on the amount of interference a device puts out and receives, which may affect the reliability of emergency communications. The amount of signal interference could also influence the decision to deploy drones; increased interference from multiple on-incident radio nets can theoretically cause drone fly-offs and malfunctions.

Lastly, SAR teams should strongly consider local weather forecasts and the likelihood that their SAR efforts will occur at night when deciding to deploy the drone-repeater system configuration. During the study, the drone was flown during the day in fair weather conditions. During testing at 2 of the 10 locations, the team experienced some moderate wind and very light rain. This did not affect the function of the drone. However, the drone used in this study would not work in high winds, steady rain, or heavy snow. It is important to be aware that flying a drone at night is illegal in most areas, likely limiting when the drone can be employed as a communication solution.

\section{Conclusions}

SAR teams should consider drones equipped with repeater systems to restore communication in rugged terrain where radios are found to be unreliable. The implementation of this solution could result in more efficient rescues and better wilderness medical outcomes at a relatively low cost. Future studies should focus on validating the cost effectiveness for the regular use of drones for SAR operations, as well as determining whether their application significantly reduces morbidity and mortality.

Acknowledgments: The authors acknowledge Nate Brooksby, Mike Thomas, Darrel Cashin with the Washington County search and rescue, Autumn Dach, Alexis Horst, and Daniel Sullivan for their helpful input in the production of this manuscript.

Author Contributions: Study concept and design (JNM, BMN, CJG, ADN); acquisition of the data (JNM, BMN); analysis of the data (JNM, BMN, CJG, APH, ADN); drafting of the manuscript (JNM, BMN, CJG, $\mathrm{APH}, \mathrm{ADN}$ ); critical revision of the manuscript (JNM, BMN, CJG, APH); and approval of final manuscript (JNM, BMN, CJG, APH, $\mathrm{ADN}$ ).

Financial/Material Support: None.

Disclosures: None.

\section{References}

1. Heggie TW, Heggie TM. Search and rescue trends associated with recreational travel in US national parks. J Travel Med. 2009;16(1):23-7.

2. Heggie TW, Heggie TM. Search and rescue trends and the emergency medical service workload in Utah's National Parks. Wilderness Environ Med. 2008;19(3):164-71.

3. Grissom CK, Thomas F, James B. Medical helicopters in wilderness search and rescue operations. Air Med J. 2006;25(1):18-25.

4. Worley GH. Wilderness communications. Wilderness Environ Med. 2011;22(3):262-9.

5. National Parks Service. 2017 annual SAR report dashboard. Vol 2017. Available at: https://nps.maps.arcgis. com/apps/opsdashboard/index.html. Accessed October $15,2019$.

6. Hung EK, Townes DA. Search and rescue in Yosemite National Park: a 10-year review. Wilderness Environ Med. 2007;18(2):111-6.

7. Visser JT, Campbell AF. New Zealand land search and rescue operations: an analysis of medical and traumatic conditions. Wilderness Environ Med. 2014;25(4):401-8. 
8. Ela GK. Epidemiology of wilderness search and rescue in New Hampshire, 1999-2001. Wilderness Environ Med. 2004;15(1):11-7.

9. Curran-Sills GM, Karahalios A. Epidemiological trends in search and rescue incidents documented by the Alpine Club of Canada from 1970 to 2005. Wilderness Environ Med. 2015;26(4):536-43.

10. Wild FJ. Epidemiology of mountain search and rescue operations in Banff, Yoho, and Kootenay National Parks, 2003-06. Wilderness Environ Med. 2008;19(4): 245-51.

11. Karaca Y, Cicek M, Tatli O, Sahin A, Pasli S, Beser MF, et al. The potential use of unmanned aircraft systems (drones) in mountain search and rescue operations. Am J Emerg Med. 2018;36(4):583-8.

12. Abrahamsen HB. A remotely piloted aircraft system in major incident management: concept and pilot, feasibility study. BMC Emerg Med. 2015;15:12.
13. Van Tilburg C. First report of using portable unmanned aircraft systems (drones) for search and rescue. Wilderness Environ Med. 2017;28(2):116-8.

14. McRae JN, Gay CJ, Nielsen BM, Hunt AP. Using an unmanned aircraft system (drone) to conduct a complex high altitude search and rescue operation: a case study. Wilderness Environ Med. 2019;30(3):287-90.

15. DJI. DJI Mavic Pro - Specs, Tutorials \& Guides - DJI. Available at: https://www.dji.com/mavic/info: DJI; 2018. Accessed October 15, 2019.

16. Federal Aviation Administration. Unmanned Aircraft Systems. Vol 2018. Updated May 25, 2018 ed. Available at: https://www.faa.gov/uas. Accessed October 15, 2019.

17. US Department of Homeland Security, 2016. National Interoperability Field Operations Guide. Washington, DC: U.S. Department of Homeland Security.

18. Singer E. Land Mobile Radio Systems. 2nd ed. Englewood Cliffs: Prentice-Hall; 2004:67-75. 